VOL. $45(1992) \quad[53-60]$

\title{
CONICAL INVERSE MAPPING THEOREMS
}

\author{
HÉLÈne FrankowsKa
}

We prove a high order conical inverse mapping theorem for set-valued maps on complete metric spaces. An application to a control problem is provided.

\section{INTRODUCTION}

Inverse mapping theorems are known to be an efficient tool in analysis of a wide range of problems. A number of them were obtained for maps between two vector spaces. However, their application in control theory is not always straightforward: they are either ill adapted to the nature of the problem or too weak.

Let us recall a functional analysis result (known under the names of Graves' or Ljusternik's theorem) stating that if a continuously differentiable map $f: X \mapsto Y$ between two Banach spaces has a surjective derivative $f^{\prime}(\bar{x})$ at some $\bar{x} \in X$, then the inverse image $f^{-1}(\cdot)$ enjoys Lipschitzian behaviour around $f(\bar{x})$.

This statement is not strong enough to answer many questions arising in Control Theory and Optimisation:

First of all, it happens quite often while dealing with control systems that the set of admissible controls is not an open subset of a Banach space. This creates a need for inverse mapping theorems on closed sets or, more generally, on metric spaces. Some problems (such as the small time controllability one) lead to set-valued maps. Finally, in many cases, the first order criterion may fail $\left(f^{\prime}(\bar{x})\right.$ is not surjective) and higher order sufficient conditions for invertibility are required. This also leads to "covering conditions" of a conical nature.

In summary there is a need for high order inverse mapping theorems for nonsmooth and even set-valued maps defined on metric spaces. This leads us to a cross-road, where a choice has to be made between

$\nearrow$ designing new tools better adapted to our purposes

$\searrow$ twisting the problems at hand to make them applicable with classical inverse mapping theorems (for instance by finding nice smooth selections.)

We shall follow the first strategy, since very fortunately one can approach set-valued maps on the same footing as single-valued ones. We refer to Set-Valued Analysis [1] for

Received 15 January 1991

Copyright Clearance Centre, Inc. Serial-fee code: 0004-9729/92 \$A2.00+0.00. 
a basic treatment of several questions dealing with set-valued maps and to [2]-[6] for higher order extensions of inverse mapping theorems. In [8] the above approach was used to prove an "angular open mapping principle" related to the shape of reachable sets for smooth affine control systems.

The aim of this paper is to prove a general Conical Inverse Mapping Theorem which, in particular, may be used to study reachable sets of a wider range of control systems than those considered in [8]. The proofs we provide here are also much simpler than those given in [4], where a less general result was obtained.

\section{Preliminaries}

To denote set-valued maps we shall use the "smooth" flush: $\leadsto$.

Consider a set-valued map $G: X \leadsto Y$ from a metric space $X$ with the metric $d_{X}$ to a normed space $Y$ with the norm $\|\cdot\|$. That is for every $x \in X, G(x)$ is a (possibly empty) subset of $Y$. The graph of $G$ is a subset of the product space $X \times Y$ given by:

$$
\operatorname{Graph}(G)=\{(x, y) \in X \times Y \mid y \in G(x)\}
$$

A set-valued map $G$ is called closed if its graph is closed in $(X \times Y, d)$.

The inverse set-valued map $G^{-1}: Y \leadsto X$ is defined by

$$
\forall y \in Y, G^{-1}(y)=\{x \in X \mid y \in G(x)\}
$$

For all $x \in X, h>0$, denote by $B_{h}(x)$ the closed ball in $X$ of center $x$ and radius $h>0$ and by $B$, the closed unit ball in $Y$. We also set $B_{\infty}(x)=X$. For a subset $L \subset X$ and $x \in X$, the distance from $x$ to $L$ is defined by

$$
\operatorname{dist}(x, L)=\inf _{y \in L} d_{X}(x, y)
$$

When $L$ is empty, then dist $(x, L)=+\infty$. Finally $G(L)$ denotes the image of $L$ by the map $G$, given by

$$
G(L)=\bigcup_{y \in L} G(y)
$$

THEOREM 1.1. Consider a closed set-valued map $G$ from a complete metric space $\left(X, d_{X}\right)$ to a normed space $Y$ and a bounded subset $K \subset Y$. Let $\left(x_{0}, y_{0}\right) \in$ $\operatorname{Graph}(G), k>0, \varepsilon>0, \delta_{z}>0, \delta_{y} \in[0,+\infty]$ be given. Assume that for some $0 \leqslant \alpha<1$ and for all $(x, y) \in G r a p h(G) \cap\left(B_{\delta_{x}}\left(x_{0}\right) \times B_{\delta_{y}}\left(y_{0}\right)\right)$,

$$
\forall h \in[0, \varepsilon], y+h^{k} K \subset G\left(B_{h}(x)\right)+\alpha h^{k} K
$$


Define $c=\sup _{y \in K}\|y\|$. Then, for every $(x, y) \in \operatorname{Graph}(G)$ and $h \in[0, \varepsilon]$, satisfying

$$
d_{X}\left(x, x_{0}\right)+h \leqslant \delta_{x} \text { and }\left\|y-y_{0}\right\|+c h^{k}(1+\alpha)\left(1-\alpha^{1 / k}\right)^{k} \leqslant \delta_{y}
$$

we have

$$
y+\left(1-\alpha^{1 k}\right)^{k} h^{k} K \subset G\left(B_{h}(x)\right) \text {. }
$$

Proof: Fix $(x, y) \in \operatorname{Graph}(G), h>0$ as in the conclusion and let $\bar{y} \in y+$ $\left(1-\alpha^{1 / k}\right)^{k} h^{k} K$. We construct $\bar{x} \in B_{h}(x)$ satisfying $\bar{y} \in G(\bar{x})$ as the limit of a sequence we shall build. Set $\left(u_{0}, v_{0}\right)=(x, y)$. By $(1)$, there exists $\left(u_{1}, v_{1}\right) \in \operatorname{Graph}(G)$ such that

$$
d_{X}\left(u_{0}, u_{1}\right) \leqslant\left(1-\alpha^{1 / k}\right) h \text { and } \bar{y} \in v_{1}+\left(\alpha^{1 / k}\left(1-\alpha^{1 / k}\right) h\right)^{k} K .
$$

Hence $\left\|v_{1}-\bar{y}\right\| \leqslant c \alpha\left(1-\alpha^{1 / k}\right)^{k} h^{k}$. Assume that we already constructed $\left(u_{i}, v_{i}\right) \in$ $\operatorname{Graph}(G), i=1, \ldots, n$ such that

$$
\begin{gathered}
d_{X}\left(u_{i-1}, u_{i}\right) \leqslant \alpha^{i-1 / k}\left(1-\alpha^{1 / k}\right) h, \\
\bar{y} \in v_{i}+\left(\alpha^{i / k}\left(1-\alpha^{1 / k}\right) h\right)^{k} K .
\end{gathered}
$$

Then

$$
d_{X}\left(x, u_{n}\right) \leqslant \sum_{i=1}^{n} d_{X}\left(u_{i-1}, u_{i}\right) \leqslant h\left(1-\alpha^{1 / k}\right) \sum_{i=0}^{n-1} \alpha^{i / k} \leqslant h
$$

Therefore $d_{X}\left(x_{0}, u_{n}\right) \leqslant \delta_{x}$ and using (3) we obtain

$$
\begin{aligned}
\left\|y_{0}-v_{n}\right\| & \leqslant\left\|y_{0}-y\right\|+\|y-\bar{y}\|+\left\|\bar{y}-v_{n}\right\| \\
& \leqslant\left\|y_{0}-y\right\|+c\left(1-\alpha^{1 / k}\right)^{k} h^{k}+c \alpha^{n}\left(1-\alpha^{1 / k}\right)^{k} h^{k} \leqslant \delta_{y} .
\end{aligned}
$$

By (3) and (1) applied to $\left(u_{n}, v_{n}\right)$, there exists $\left(u_{n+1}, v_{n+1}\right) \in \operatorname{Graph}(G)$ satisfying (2) and (3) with $i=n+1$.

Inequality (2) implies that $\left\{u_{i}\right\}$ is a Cauchy sequence converging to some $\bar{x}$ and inclusion (3) implies that $\lim _{i \rightarrow \infty} v_{i}=\bar{y}$. Since $\operatorname{Graph}(G)$ is closed, $\bar{y} \in G(\bar{x})$. Moreover, by taking the limit in (4), we obtain $d_{X}(x, \bar{x}) \leqslant h$.

\section{Conical inverse Mapping Theorem}

Let $T$ be a metric space and $\left\{A_{\tau}\right\}_{\tau \in T}$ be a family of subsets of a metric space $X$. The lower limit Lim inf of $A_{\tau}$ at $\tau_{0} \in T$ are closed sets defined by

$$
\operatorname{Lim} \inf _{\tau \rightarrow \tau_{0}} A_{\tau}=\left\{v \in X \mid \lim _{\tau \rightarrow \tau_{0}} \operatorname{dist}\left(v, A_{\tau}\right)=0\right\} .
$$


Definition 2.1: [4] Consider $(x, y) \in \operatorname{Graph}(G), k>0$. The $k$-th order vatiation of $G$ at $(x, y)$ is the closed subset of $Y$ defined by

$$
G^{k}(x, y):=\operatorname{Lim} \inf _{\substack{\left.z^{\prime}, y^{\prime}\right) \rightarrow G(x, y) \\ h \rightarrow 0+}} \frac{G\left(B_{h}\left(x^{\prime}\right)\right)-y^{\prime}}{h^{k}}
$$

where $\rightarrow G$ denotes the convergence in $\operatorname{Graph}(G)$.

In other words $v \in G^{k}(x, y)$ if and only if for all sequences $h_{i}>0,\left(x_{i}, y_{i}\right) \in$ $\operatorname{Graph}(G)$, converging to zero and $(x, y)$ respectively, there exists a sequence $v_{i} \in Y$ such that

$$
\forall i \geqslant 1, \quad y_{i}+h_{i}^{k} v_{i} \in G\left(B_{h_{i}}\left(x_{i}\right)\right), \quad \lim _{i \rightarrow \infty} v_{i}=v .
$$

Properties of variations are summarised in the following theorem.

ThEorem 2.2. [4] Let $(x, y) \in \operatorname{Graph}(G), k>0$. Then:

(i) For all $K>k, \mathbf{R}_{+} G^{k}(x, y) \subset G^{K}(x, y)$. In particular, the sequence $\left\{G^{k}(x, y)\right\}_{k>0}$ is nondecreasing.

(ii) For all $\lambda_{i} \geqslant 0, v_{i} \in G^{k}(x, y), i=0, \ldots, m$ with $\sum_{i=0}^{m} \lambda_{i}=1$,

$$
\sum_{i=0}^{m} \lambda_{i}^{k} v_{i} \in G^{k}(x, y)
$$

Thus $G^{1}(x, y)$ is convex and $G^{k}(x, y)$ is starshaped at zero.

(iii) If $k \geqslant 1$, then co $\left(G^{k}(x, y)\right) \subset \mathbf{R}_{+} G^{k}(x, y)$. Furthermore, if the dimension of $Y, \operatorname{dim}(Y)$, is finite, then

$$
\overline{c o}\left(G^{k}(x, y)\right) \subset(\operatorname{dim}(Y)+1)^{k-1} G^{k}(x, y) .
$$

(iv) $\bigcup_{\lambda \geqslant 0} \lambda G^{k}(x, y)=Y \Longleftrightarrow 0 \in \operatorname{Int}\left(c o\left(G^{k}(x, y)\right)\right)$. Moreover, if $Y$ is finite dimensional, then

$$
\bigcup_{\lambda \geqslant 0} \lambda G^{k}(x, y)=Y \Longleftrightarrow 0 \in \operatorname{Int}\left(G^{k}(x, y)\right)
$$

which is also equivalent to

$\exists v_{1}, \ldots, v_{p} \in G^{k}(x, y)$ such that $0 \in \operatorname{Int}\left(\operatorname{co}\left\{v_{1}, \ldots, v_{p}\right\}\right)$ 
TheOREM 2.3. Let $X$ be a complete metric space, $G: X \leadsto \mathbf{R}^{n}$ be a closed set-valued map and $\bar{x} \in X, \bar{y} \in G(\bar{x}), k>0$. Consider a nonempty convex compact subset $Q \subset \operatorname{Int}\left(G^{k}(\bar{x}, \bar{y})\right)$ and $0<\gamma<1$.

Then for all $(x, y) \in \operatorname{Graph}(G)$ near $(\bar{x}, \bar{y})$ and all $h>0$ small enough,

$$
y+\gamma h^{k} Q \subset G\left(B_{h}(x)\right) \text {. }
$$

Furthermore, if

$$
m:=\inf _{0 \neq q \in Q} \sup _{q^{\prime} \in\left(\mathbb{R}_{+q}\right) \cap Q}\left\|q^{\prime}\right\|>0
$$

then for all $\left(x_{1}, y_{1}\right) \in \operatorname{Graph}(G)$ near $(\bar{x}, \bar{y})$ and all $y_{2}$ near $\bar{y}$ satisfying $y_{2} \in y_{1}+\mathbf{R}_{+} Q$ we have

$$
\operatorname{dist}\left(x_{1}, G^{-1}\left(y_{2}\right)\right) \leqslant(\gamma m)^{-1 / k}\left\|y_{1}-y_{2}\right\|^{1 / k} .
$$

Proof: Let $0<\lambda<1$ be such that $\gamma=\left(1-(1-\lambda)^{1 / k}\right)^{k}$ and $\varepsilon>0$ be such that $Q+\varepsilon B \subset G^{k}(\bar{x}, \bar{y})$. By Theorem 2.2 (ii) the set

$$
Q_{1}:=\lambda(Q+\varepsilon B) \subset G^{k}(\bar{x}, \bar{y}) .
$$

Fix $v \in Q_{1}$. Then there exists $\delta_{v}>0$ such that for all $0 \leqslant h \leqslant \delta_{v}$ and $(x, y) \in$ $\operatorname{Graph}(G)$ satisfying $d_{X}(x, \bar{x}) \leqslant \delta_{v},\|y-\bar{y}\| \leqslant \delta_{v}$ we have

$$
y+h^{k} v \in G\left(B_{h}(x)\right)+\frac{\varepsilon \lambda}{2}(1-\lambda) h^{k} B .
$$

Hence for all $w \in Q_{1}$ such that $\|w-v\|<\bar{\varepsilon}:=\varepsilon \lambda(1-\lambda) / 2$ and all $x, y, h$ as above we have

$$
y+h^{k} w \in G\left(B_{h}(x)\right)+\varepsilon \lambda(1-\lambda) h^{k} B .
$$

Since $Q_{1}$ is compact, there exist $v_{i} \in Q_{1}, i=1, \ldots, m$ such that

$$
Q_{1} \subset \bigcup_{i=1}^{m}\left\{w \in \mathbf{R}^{n} \mid\left\|w-v_{i}\right\|<\bar{\varepsilon}\right\} .
$$

Let $\delta=\min _{i=1, \ldots, m} \delta_{v_{1}}$ and consider $0 \leqslant h \leqslant \delta$ and $(x, y) \in \operatorname{Graph}(G)$ with $d_{X}(x, \bar{x}) \leqslant \delta,\|y-\bar{y}\| \leqslant \delta$. Fix $w \in Q_{1}$ and let $1 \leqslant i \leqslant m$ be such that $\left\|w-v_{i}\right\|<\bar{\varepsilon}$. Then, by the choice of $\delta,(7)$ holds true. Since $w \in Q_{1}$ is arbitrary we proved that

$$
y+h^{k} \lambda(Q+\varepsilon B) \subset G\left(B_{h}(x)\right)+\varepsilon \lambda(1-\lambda) h^{k} B .
$$


Adding $(1-\lambda) h^{k} Q$ to both sides of the above inclusion and using that $Q$ is convex we get

$$
y+h^{k}(Q+\varepsilon \lambda B) \subset G\left(B_{h}(x)\right)+(1-\lambda) h^{k}(Q+\varepsilon \lambda B) .
$$

Applying Theorem 1.1 we deduce that for all $(x, y) \in \operatorname{Graph}(G)$ near $(\bar{x}, \bar{y})$ and all $h>0$ sufficiently small

$$
y+\gamma h^{k} Q \subset y+\left(1-(1-\lambda)^{1 / k}\right)^{k} h^{k}(Q+\varepsilon \lambda B) \subset G\left(B_{h}(x)\right) .
$$

To prove the second statement fix $\left(x_{1}, y_{1}\right) \in \operatorname{Graph}(G)$ near $(\bar{x}, \bar{y})$ and $y_{2} \in y_{1}+\mathbf{R}_{+} Q$ sufficiently close to $\bar{y}$. It is enough to consider the case $y_{2} \neq y_{1}$. Then for some $h>0$ and $q \in Q$ different from zero, $y_{2}=y_{1}+\gamma h^{k} q$. By (6) we may assume that $\|q\| \geqslant m$. By the first statement $y_{2} \in G\left(B_{h}\left(x_{1}\right)\right)$. Consequently,

$$
\text { dist }\left(x_{1}, G^{-1}\left(y_{2}\right)\right) \leqslant h=(\gamma\|q\|)^{-1 / k}\left\|y_{1}-y_{2}\right\|^{1 / k}
$$

The above theorem yields the following "conical open mapping principle".

Corollary 2.4. (Conical Open Mapping Principle) Let $X$ be a complete metric space, $G: X \leadsto \mathbf{R}^{n}$ be a closed set-valued map and $\bar{x} \in X, \bar{y} \in G(\bar{x}), k>0$. Consider a nonempty convex compact subset $Q \subset \operatorname{Int}\left(G^{k}(\bar{x}, \bar{y})\right)$ and $0<\gamma<1$. Then for all $(x, y) \in \operatorname{Graph}(G)$ near $(\bar{x}, \bar{y})$ and all $h>0$ small enough

$$
y+[0, \gamma] h^{k} Q \subset G\left(B_{h}(x)\right)
$$

Proof: By Theorem 2.3 for all $(x, y) \in \operatorname{Graph}(G)$ near $(\bar{x}, \bar{y})$ and all $h>0$ small enough

$$
y+\gamma h^{k} Q \subset G\left(B_{h}(x)\right) .
$$

Hence for every $0 \leqslant \lambda \leqslant 1$ we have

$$
y+\gamma \lambda^{k} h^{k} Q \subset G\left(B_{\lambda h}(x)\right) \subset G\left(B_{h}(x)\right) .
$$

\section{Application: Geometry of Reachable sets}

Let $U$ be a complete separable metric space and $f: \mathbf{R}^{n} \times U \mapsto \mathbf{R}^{n}$ be a continuous function, $x_{0} \in \mathbf{R}^{n}$. We assume that

(a) $\exists \varepsilon>0, l>0$ such that $\forall u \in U, f(\cdot, u)$ is $l$-Lipschitz on $B_{\varepsilon}\left(x_{0}\right)$;

(b) for some $\bar{u} \in U, f\left(x_{0}, \bar{u}\right)=0$, that is, $x_{0}$ is an equilibrium;

(c) for all $x$ near $x_{0}, f(x, U)$ is a convex, compact subset of $\mathbf{R}^{n}$. 
Consider the control system

$$
\left\{\begin{array}{l}
x^{\prime}=f(x, u(t)), u \in \mathcal{U}_{T}, T>0 \\
x(0)=x_{0}
\end{array}\right.
$$

where $\mathcal{U}_{T}$ denotes the set of measurable maps $u:[0, T] \mapsto U$.

An absolutely continuous function $x \in W^{1,1}(0, T)$ (the Sobolev space) is called a solution to the control system (8) if $x(0)=x_{0}$ and there exists $u \in \mathcal{U}_{T}$ such that $x^{\prime}(t)=f(x(t), u(t))$ almost everywhere in $[0, T]$.

For all $T>0$ the reachable set of the system (8) at time $T$ is given by

$$
R(T)=\left\{x(T) \mid x \in W^{1,1}(0, T) \text { is a solution to }(8)\right\} \text {. }
$$

The following notion was introduced in [2] to study small time local controllability.

Definition 3.1: A vector $v \in \mathbf{R}^{n}$ is called a variation of $R(\cdot)$ at zero of order $k \geqslant 1$ if for all $t \geqslant 0$

$$
x_{0}+t^{k} v \in R(t)+o\left(t^{k}\right)
$$

The set of all variations of order $k \geqslant 1$ at zero is denoted by $\mathcal{V}_{k}$.

ThEOREM 3.2. [2, 7] Under the above assumptions, $\mathcal{V}_{k}=R^{k}\left(0, x_{0}\right)$. Consequently $\overline{c o}\left(\mathcal{V}_{k}\right) \subset(n+1)^{k-1} \mathcal{V}_{k}$.

Hence Theorem 2.3 yields

THEOREM 3.3. Let $k \geqslant 1$ and $Q$ be a nonempty convex compact subset of the set $\operatorname{Int}\left(\mathcal{V}_{k}\right)$ not containing zero. Then for some $M>0, \varepsilon>0$, for all small $t \geqslant 0$, for all $x \in R(t)$ and $y \in B_{\varepsilon}\left(x_{0}\right)$ satisfying $y \in x+\mathbf{R}_{+} Q$ we have

$$
y \in R\left(t+M\|x-y\|^{1 / k}\right) \text {. }
$$

The following corollary extends the result of [8].

Corollary 3.4. Let $\mathcal{C}$ denote the (convex) cone spanned by $\mathcal{V}_{k}$ and $K$ be a closed convex cone which does not contain any whole line such that $K \backslash\{0\} \subset \operatorname{Int}(\mathcal{C})$. Then there exist $\delta>0, M>0$ such that for all small $t \geqslant 0$ and all $x \in R(t)$ we have

$$
x+K \cap \delta B \subset R\left(t+M \delta^{1 / k}\right) \text {. }
$$

Proof: Since $K \backslash\{0\} \subset \operatorname{Int}(\mathcal{C})$ and $\mathcal{C}$ is spanned by the set $\overline{c o}\left(\mathcal{V}_{k}\right)$ containing zero, there exists $\varepsilon>0$ such that $K \cap \varepsilon B \subset \overline{c o}\left(\mathcal{V}_{k}\right)$. Hence

$$
Q_{1}:=\overline{c o}\{x \in K \mid\|x\|=\varepsilon\} \subset \overline{c o}\left(\mathcal{V}_{k}\right) \text {. }
$$

On the other hand, the cone spanned by $Q_{1}$ is equal to $K$. Since $K \backslash\{0\} \subset \operatorname{Int}(\mathcal{C})$, we have $Q_{1} \subset$ Int $\overline{c o}\left(\mathcal{V}_{k}\right)$. Using the assumption that $K$ does not contain any whole line, we deduce that $0 \notin Q_{1}$. By Theorem 2.2 we have $Q:=(n+1)^{1-k} Q_{1} \subset \operatorname{Int}\left(\mathcal{V}_{k}\right)$. Theorems 3.2 and 3.3 end the proof. 


\title{
REFERENCES
}

[1] J-P. Aubin and H. Frankowska, Set-valued analysis (Birkhäuser, Boston, Basel, Berlin, 1990).

[2] H. Frankowska, 'Théorème d'application ouverte pour des correspondances', ComptesRendus de l'Académie des Sciences Série 1302 (1986), 559-562.

[3] H. Frankowska, 'Théorèmes d'application ouverte et de fonction inverse', Comptes-Rendus de l'Académie des Sciences 305 (1987), 773-776.

[4] H. Frankowska, 'An open mapping principle for set-valued maps', J. Math. Anal. Appl. 127 (1987), 172-180.

[5] H. Frankowska, 'High order inverse function theorems', Proceedings of the Conference on Nonlinear Analysis and Applications, Perpignan, June, 1987, in Analyse Non Linéaire, Editors Attouch, Aubin, Clarke, Ekeland, pp. 283-304 (Gauthier-Villars \& C.R.M., Université de Montréal, 1989).

[6] H. Frankowska, 'Some inverse mapping theorems', Ann. Inst. H. Poincaré Anal. Non Linéaire 7 (1990), 183-234.

[7] H. Frankowska, 'Local controllability of control systems with feedbacks', J. Optim. Theory Appl. 60 (1989), 277-296.

[8] M. Kawski, 'An angular open mapping principle', Proceedings of the 8th International Conference on Analysis and Optimization of Systems, in Lecture Notes in Control and Information Sciences, pp. 361-371 (Springer Verlag, Berlin, Heidelberg, New York, 1988).

\author{
Ceremade \\ Université de Paris-Dauphine \\ 75775 Paris Cedex 16 \\ France
}

